\title{
All-Optical Computing Based on Convolutional Neural Networks
}

\section{Kun Liao}

Department of Physics, Peking University

\section{Ye Chen}

Department of Physics, Peking University

\section{Zhongcheng Yu}

Department of Physics, Peking University

Xiaoyong Hu ( $\nabla$ xiaoyonghu@pku.edu.cn )

Department of Physics, Peking University

\section{Xingyuan Wang}

Beijing University of Chemical Technology

\section{Cuicui Lu}

Beijing Institute of Technology

Hongtao Lin

Zhejiang University

\section{Qingyang Du}

Massachusetts Institute of Technology

\section{Juejun Hu}

Massachusetts Institute of Technology

\section{Qihuang Gong}

Peking University

\section{Article}

Keywords: all-optical computing, convolutional neural networks, silicon waveguide

Posted Date: August 20th, 2020

DOl: https://doi.org/10.21203/rs.3.rs-59249/v1

License: (9) This work is licensed under a Creative Commons Attribution 4.0 International License. Read Full License 
Version of Record: A version of this preprint was published at Opto-Electronic Advances on January 1st, 2021. See the published version at https://doi.org/10.29026/oea.2021.200060. 


\section{All-Optical Computing Based on Convolutional Neural Networks}

\section{Kun Liao ${ }^{1}$, Ye Chen ${ }^{1}$, Zhongcheng Yu ${ }^{1}$, Xiaoyong Hu ${ }^{1,2^{*}}$, Xingyuan Wang ${ }^{3 *}$, Cuicui $\mathbf{L u}^{4}$, Hongtao Lin ${ }^{5^{*}}$, Qingyang $\mathrm{Du}^{6}$, Juejun $\mathrm{Hu}^{6}$, Qihuang Gong ${ }^{1,2}$}

${ }^{1}$ State Key Laboratory for Mesoscopic Physics \& Department of Physics, Collaborative Innovation Center of Quantum Matter, Beijing Academy of Quantum Information Sciences, Nano-optoelectronics Frontier Center of Ministry of Education, Peking University, Beijing 100871, China

${ }^{2}$ Collaborative Innovation Center of Extreme Optics, Shanxi University, Taiyuan, Shanxi 030006, China

${ }^{3}$ College of Mathematics and Physics, Beijing University of Chemical Technology, Beijing 100029, China

${ }^{4}$ Beijing Key Laboratory of Nanophotonics and Ultrafine Optoelectronic Systems, School of Physics, Beijing Institute of Technology, Beijing 100081, China

${ }^{5}$ College of Information Science \& Electronic Engineering, Zhejiang University, Hangzhou 310027, China

${ }^{6}$ Department of Materials Science and Engineering, Massachusetts Institute of Technology, Cambridge, MA 02139, USA

*Email: xiaoyonghu@pku.edu.cn; wang_xingyuan@mail.buct.edu.cn; hometown@zju.edu.cn 


\section{Abstract}

The rapid development of information technology has fueled an ever-increasing demand for ultrafast and ultralow-energy-consumption computing. Existing computing instruments are predominantly electronic processors, which use electrons as information carriers and possess von Neumann architecture featured by physical separation of storage and processing. The scaling of computing speed is limited not only by data transfer between memory and processing units, but also by RC delay associated with integrated circuits. Moreover, excessive heating due to Ohmic losses is becoming a severe bottleneck for both speed and power consumption scaling. Using photons as information carriers is a promising alternative. Owing to weak third-order optical nonlinearity of conventional materials, building integrated photonic computing chips has been a challenge. Here, we report a strategy to realize ultrafast and ultralow-energyconsumption all-optical computing based on convolutional neural networks, leveraging entirely linear optical interactions. The device is constructed from cascaded silicon Y-shaped waveguides with side-coupled silicon waveguide segments which we termed "weight modulators" to enable complete phase and amplitude control in each waveguide branch. The generic device concept can be used for equation solving, multifunctional logic operation, Fourier transformation, series expanding and encoding, as well as many other mathematical operations. Multiple computing functions including transcendental equation solvers, multifarious logic gate operators, and half-adders were experimentally demonstrated to validate the all-optical computing performances. The time-of-flight of light through the network structure corresponds to an ultrafast computing time of the order of several picoseconds with an ultralow energy consumption of dozens of femtojoules per bit. Our approach can be further 
expanded to fulfill other complex computing tasks based on non-von Neumann architectures and thus paves a new way for on-chip all-optical computing.

\section{Introduction}

The demand for ultrahigh-speed and energy-efficient computing ${ }^{1}$ has been increasing exponentially driven by the rapid development of advanced engineering calculations, economic data analysis, and cloud computing. Traditional electronic processors, the pre-dominant computing platform to date, adopt the von Neumann architecture ${ }^{2,3}$ where storage and processing units are physically separated. In von Neumann processors, limited data communication bandwidth between the memory and processing units as well as RC delay of integrated circuits have become major barriers towards continuing scaling of computing speed $^{4,5}$. Moreover, heat dissipation due to resistive losses in electrical wires severely compromises energy efficiency of traditional electronic processors ${ }^{6}$. These limitations make it difficult to realize high speed and low energy consumption simultaneously ${ }^{7,8}$. Specialized processors, such as graphic process units designed for mathematical calculation tasks and field programmable gate arrays specialized for arithmetic logic operations, utilize modified Von Neumann architecture ${ }^{5,9}$, which however still suffer from the speed and energy consumption limitations. All-optical computing adopting photons as information carriers offers a promising alternative approach. To date, optical computing usually relies on third-order optical nonlinearity to implement all-optical control, which requires ultrafast and giant third-order optical nonlinearity in photonic materials ${ }^{10,11}$. However, ultrafast response time and giant nonlinearity often presents an inherent trade-off in optical materials such that larger nonlinear susceptibility can only be attained at the expense of slower response time. The trade-off imposes 
a major challenge to constructing integrated photonic processors following the von Neumann architecture, often mandating complicated heterogeneous integration of various photonic devices in a single chip. Hence, exploring new architectures and unconventional computing schemes for all-optical computing becomes imperative.

Here, we report a new strategy to realize ultrafast and ultralow-energy-consumption alloptical computing including equation solving, multifunctional logic operation, Fourier transformation, series expanding and encoding based on optical convolutional neural network $(\mathrm{CNN})$ without resorting to optical nonlinearity. Inspired by biological brains ${ }^{12,13}$, optical neural networks have been used to carry out image classification ${ }^{14,15}$, speech recognition ${ }^{16}$, and selflearning $\operatorname{tasks}^{17}$. For the first time, we propose an all-optical computing chip based on physically-fixed CNN. Optical CNNs possesses a non-von Neumann architecture, which underlies its ultrafast computing time and ultralow energy consumption. They are able to perform convolutional calculation using matrix multiplication without the aid of optical nonlinearity, conducive to multi-tasking processing and significantly reduced energy consumption $^{18,19}$. Furthermore, owing to their powerful prediction capability, a single network can solve a specific class of computing problems rather than one single task. This scheme has built a new platform for all-optical computing, on which almost all signal processing functions are allowed to be implemented.

The optical CNN consists of cascaded silicon Y-shaped waveguides with side-coupled silicon waveguide segments designed to control the amplitude and phase of light in the waveguide branches. This conceptually and architecturally simple design solely capitalizes on linear optical interactions, and hence uniquely affords both ultrafast computing time and low 
energy consumption. Importantly, the design is also scalable to handle CNNs with arbitrary network complexity. Our scalable optical $\mathrm{CNN}$ architecture presents a universal platform for implementing CNN-related functions leveraging the vast asset base of algorithms that have been matured in the field of computer research (Supplementary Material Section I). Another important advantage of CNNs is that they can protect signals from distortion compared with fully-connected neural networks, as CNNs only contain local connections. As a proof-ofconcept, we experimentally implemented the network design through several computation tasks including transcendental equations solvers, multifunctional logic gate operators, and halfadders.

\section{Scalable network configuration}

To realize CNNs in an on-chip platform, we designed an all-optical network to emulate the convolutional operations (as shown in Fig. 1a). The signals fed into the network are encoded in the form of light amplitude distribution in discrete input waveguides. The network weights optimized to yield the target solutions are implemented through convolution operation between layers, i.e. $\tilde{X}^{l}=\widetilde{W}_{l, l-1} \tilde{X}^{l-1}$, where $\tilde{X}^{l}$ and $\tilde{X}^{l-1}$ represent the light amplitude distributions in the $l^{\text {th }}$ and $(l-1)^{\text {th }}$ layers, respectively, and $\widetilde{W}_{l, l-1}$ gives the weight that dictates the way signals are passed from the $l^{\text {th }}$ layer to the $(l-1)^{\text {th }}$ layer. Results of the computing tasks are given in the form of light amplitude distribution in a set of discrete output waveguides. To the best of our knowledge, this is the first implementation of a physically-fixed CNN on an all-optical chip.

The $\mathrm{CNN}$ is constructed from cascaded element structures comprising Y-shaped silicon waveguides side-coupled with silicon weight modulators. As an example, the schematic structure of the all-optical transcendental equation solver based on CNN is shown in Fig. $1 \mathrm{~b}$. 
There are three layers of the element structure arrays. Each element structure connected to two adjacent element structures in the adjacent layers. Weight modulators are used to regulate the weights of the network according to the coupled mode theory. The weight modulator waveguide (as shown in Fig. 2a) has the same width as the transmission waveguide to ensure efficient coupling and large amplitude modulation. As Fig. $2 \mathrm{~b}$ shows, the magnitude of weight $w$, which stands for the amplitude transmittance of the signal light in the transmission waveguide within the element structure, can be continuously tuned from 0.025 to 0.955 by varying the length $(a)$ of weight modulator and the gap width $(b)$ between the transmission waveguide and the weight modulator. Similarly, the phase of weight $w$ representing the phase of the signal light at the waveguide output port can be continuously adjusted from 0 to $2 \pi$ by changing $a$ and $b$ as well (as shown in Fig. 2c). Importantly, the amplitude and phase of weight $w$ can be independently adjusted to achieve arbitrary control of the signal light. For further discussions of the weight modulation method, please see Supplementary Material Section II.

It's worth mentioning that other complex mathematical operations can be systematically designed into the unified optical $\mathrm{CNN}$ architecture by cascading the Y-shaped element structures. In the followings, we elaborate several examples of our optical CNN design being implemented as transcendental equation solvers, multifarious logic gate operators, and halfadders.

\section{All-optical transcendental equation solver}

Since equations are effective tools for describing system states and processes, solving equations ${ }^{20}$ can inform the state of the system under investigation and predict the trajectory of system evolution. Since transcendental equations can only be numerically solved except for a 
few cases, numerical solution of transcendental equations is still an important subject in mathematical calculations. We have developed a solver that can predictably solve transcendental equations using optical CNN with exceptional computational performance.

A transcendental equation with the form of a trigonometric function is selected because any arbitrarily complex mathematical expressions can be decomposed into trigonometric functions by Fourier decomposition, which means that we can solve any other transcendental equations in principle. The all-optical transcendental equation solver is used to solve the equation with a variable parameter $k$ :

$$
\cos (2 k x)+4=\tan (k x)
$$

We choose to represent the input waveform (in this case $\cos (2 k \mathrm{x})+4)$ by encoding its values at a set of evenly spaced $x$ points with light amplitudes in a waveguide array. Firstly, light from a single input waveguide is passed through a 5-layer cascaded Y-branch structures to generate the discretized waveform of $\cos (2 k \mathrm{x})+4$ in the range from 3 to $3+8 \times i / \mathrm{N}$, where $i$ is an integer in the range from 0 to 29 , and $\mathrm{N}$ is the total number of waveguides. Then the output signal representing the discretized waveform is input to the $\mathrm{CNN}$ with a total layer number of 3. An SEM image of this network structure is shown in Fig. 1c. The fixed network weights were pre-determined prior to fabrication via an iterative training algorithm detailed in Supplementary Material Section III, and implemented using the silicon weight modulator structures in this optical CNN. The network weights are optimized such that the solution to the equation is given by the position of the waveguide yielding the maximum output intensity:

$$
x=3+8 \times i^{\prime} / N^{\prime}
$$

where $i^{\prime}$ is an integer, and $N^{\prime}$ is the total number of output waveguides. To validate the 
performance of the optical CNN equation solver, solution of Eq. 1 is performed by inputting a series of waveforms with the parameter $k$ equaling to $1.67,1.84$ and 2.35 , respectively. Fig. 3a shows the optical CNN output for $k=1.67$, and results pertaining to the other two $k$ values are presented in Supplementary Material Section III. Here, we define the deviation of the CNN output solution from the true solution as:

$$
\frac{x_{\text {exp }}-x_{\text {theor }}}{X_{\text {out }}}
$$

where $x_{\text {exp }}$ and $x_{\text {theor }}$ correspond to the encoded $x$ values associated with the maximum intensity waveguide (following Eq. 2), and the subscripts exp and theor denote the experimentally measured and theoretically predicted results, respectively. $X_{\text {out }}$ represents the entire range of $x$ over which the solution is sought ( 3 to $3+8 \times 26 / 27$ in this case), which is dictated by the total number of output waveguides $N^{\prime}$ (27 in our device). Fig. $3 \mathrm{~b}$ summarizes the solution deviations for the three $k$ values. The test result shows that our transcendental equation solver has achieved high accuracy with a maximum deviation less than $5 \%$, and in most cases the deviations are less than 3\%. The deviation results from the finite number of output waveguides and imperfect sample fabrication. Thus, it should be emphasized that the accuracy of the solution can be improved by increasing the number of output waveguides in theory.

Besides excellent solution accuracy, the all-optical equation solver also features ultrafast and energy-efficiency computation. The total computing time, characterized by the time-offlight of light through the entire structure (including the waveform discretization section), is 9.4 ps, and the effective operation time of the 3-layer $\mathrm{CNN}$ is as short as $1.3 \mathrm{ps}$. The optical solver can also claim ultralow energy consumption. In our experiments, the computation energy 
overhead is $92 \mathrm{fJ} /$ bit based on the laser pulse power we used. Our analysis further demonstrated that the shot-noise-limited mean error converges to a limit bounded by the discreteness of the network output at pulse energies above a few aJ/bit. (Supplementary Material Section VI).

The optical CNN architecture presented here also offers the unique potential of crosstalk elimination. Crosstalk in optical analog computing is generally caused by light backscattering between adjacent layers in a densely integrated platform. Based on our device design, the crosstalk is expected to be naturally eliminated by means of the error back propagation optimization process. Stability analysis of our network further demonstrates its high fault tolerance to defects such as weight deviation and waveguide damage (Supplementary Material Section V).

\section{Multifarious logic gate operators}

All-optical logic gates constitute the basic building blocks for ultra-high-speed all-optical chips, as any complex optical logic circuit can be composed of these logic gates. In addition, logic operation sets the foundation for more complex optical signal processing functions, such as addressing $^{21}$, data coding ${ }^{22}$, parity checking ${ }^{23}$ and signal extraction ${ }^{24}$. However, current alloptical logic device designs based on linear coherence of signal light or nonlinear interactions still face challenges in realizing reconfigurability and multifunctional operation (implementing multiple logic functions in a single chip) with high speed and low power consumption.

We leverage the scalability of our network to optimize on-chip all-optical multifarious logic devices. The design optimizes 6 input ports, including 2 signal input terminals and 4 control bits with a total of 5 layers (as shown in Figs. 4a,b). Similar to the all-optical equation 
solver, the fixed network weights were optimized using the iterative algorithm. Sixteen logic functions (representing exhaustive combinations of output results corresponding to all four possible input signals $11,10,01$, and 00 is $2^{4}=16$ ) can be realized through seven different CNN structures, each with different network weights and responds to a different set of control bits. Each structure can perform 3 to 4 logic functions. Here we illustrate one of the optical CNN structures in Fig. 4a, and characterization results of the other six structures are elaborated in Supplementary Material Section IV. As we can see from Fig. 4c, when the control bits are 1001, 0110 and 1010, the optical CNN performs " $A+B(\mathrm{OR})$ ", " $A \odot B(\mathrm{XNOR})$ ", and " $(A+$ $\bar{A}) B$ " functions respectively. The intensity contrast of logic states 0 and 1 are experimentally measured as $7.2 \mathrm{~dB}, 10.4 \mathrm{~dB}$, and $12.9 \mathrm{~dB}$ respectively for the three functions. The time-offlight computing time is 3.3 ps with an energy consumption of $71 \mathrm{fJ} / \mathrm{bit}$. Our analysis further demonstrated that energy consumption down to $10.4 \mathrm{aJ} /$ bit can be achieved while maintaining a low error rate of $10^{-9}$ (Supplementary Material Section VI). In Fig. 4d, the optical CNN responses when performing the three logic functions are overlaid in one plot, showing a minimal output optical intensity contrast between the logic states 0 and 1 of $4.9 \mathrm{~dB}$. The result shows that the optical CNN's output logic states are readily distinguishable while performing multiple logic functions.

\section{Half-adder}

All-optical half-adder can perform the calculation task of adding two input data bits and yielding a Sum bit and a Carry bit in an all-optical implementation (Fig. 5a). Half-adder is a basic unit of arithmetic logic operation optical circuits: for example, a full-adder can be realized by cascading two half-adders. Here we demonstrate an all-optical half-adder based on our 
optical CNN platform. 12 network weights are determined through algorithm optimization, and an SEM image of half-adder is shown in Fig. 5b. The arithmetic logic operations of "1" + "1" $=($ Sum “0”, Carry “1”), “0” + “1” = (Sum "1", Carry “0”), and "1" + “0” = (Sum "1", Carry “ 0 ”) are realized. The average optical intensity contrast between logic states 0 and 1 is $14.2 \mathrm{~dB}$ (Fig. 5c). The time-of-flight computing time is 2.7 ps with an energy consumption of 50.8 fJ/bit. Similarly, our analysis further demonstrated that energy consumption down to $23.8 \mathrm{aJ} / \mathrm{bit}$ can be achieved while maintaining a low error rate of $10^{-9}$ (Supplementary Material Section VI). The function of the half-adder is successfully demonstrated while achieving high intensity contrast, which further validates that the CNN design is highly scalable and broadly applicable to a wide variety of all-optical processing functions.

Moreover, based on this element structure, the desired phase distribution can be obtained at output ports by adjusting weights of network, then the spatial filtering system can be constructed to realize the Fourier transformation of the input signal. Similarly, the input function can be expressed as a linear combination of multiple monomials at a given point to achieve series expansion. In addition, by defining the input-output relationship in advance to realize the network training, the output signals corresponding to different input signals are specified to represent specific code groups. Thus the encoder is implemented. From above, almost all signal processing functions are allowed to be implemented on the proposed platform, which promotes the whole field of nanophotonics. The performance benchmark and significance of this work are presented in Supplementary Material Section VII.

\section{Conclusion}

In this paper, we experimentally demonstrated the first physically-fixed CNN for all-optical 
computing based on silicon waveguides. Unlike prior work resorting to nonlinear interactions, our optical CNN entirely relies on linear optical components and is formed by cascading a simple, universal element structure comprising Y-shaped silicon waveguides side-coupled with silicon weight modulators. We implemented the design to realize all-optical transcendental equation solvers, multifarious logic gate operators, and half-adders, all of which exhibit picosecond-scale ultrafast operation and ultralow energy consumption of the order of tens of femtojoules per bit. This optical network architecture is readily scalable can be further extended to execute other complex computing tasks simply by cascading the basic element structures. Furthermore, this platform offers the possibility of parallel computing using wavelength multiplexing. Our work therefore points to a promising direction for next-generation all-optical computing systems. 


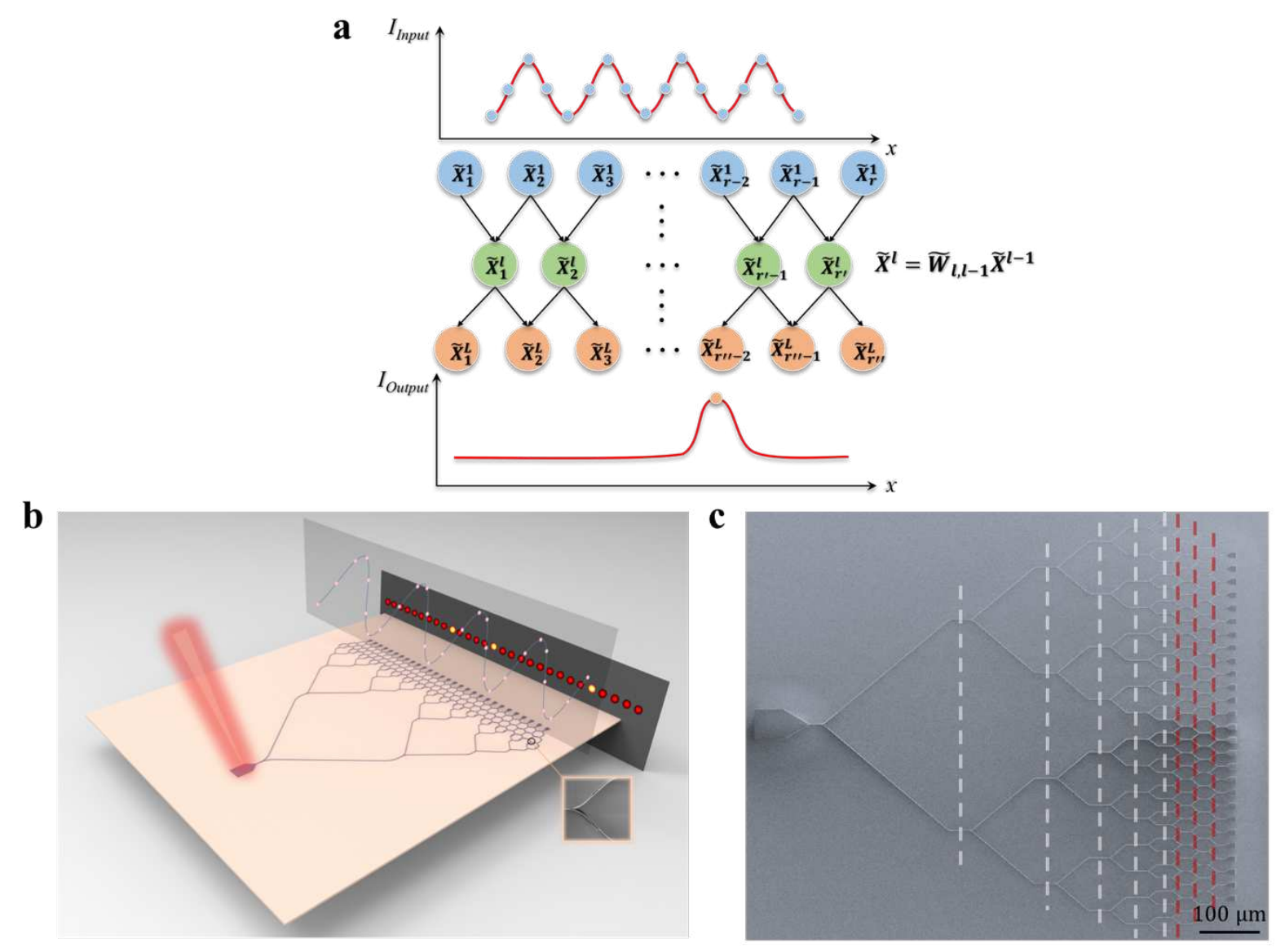

Figure 1 | General architecture of the all-optical computing framework. a, The CNN

architecture showing the connections between adjacent layers: $\tilde{X}^{l}=\widetilde{W}_{l, l-1} \tilde{X}^{l-1}$, where $\tilde{X}^{l}$ and $\tilde{X}^{l-1}$ represent the $l^{\text {th }}$ and $(l-1)^{\text {th }}$ layer's optical signal respectively, $\widetilde{W}_{l, l-1}$ shows the weight that determines how signals are propagated from $l^{\text {th }}$ layer to $(l-1)^{\text {th }}$ layer. $\mathbf{b}$, A schematic diagram of the all-optical transcendental equation solver. c, Top-view SEM image of the alloptical transcendental equation solver, where the scale bar is $100 \mu \mathrm{m}$. Here, the white dotted lines mark the five layers for waveform discretization, and the red dotted lines separate the three layers of the optical CNN structure. 
b

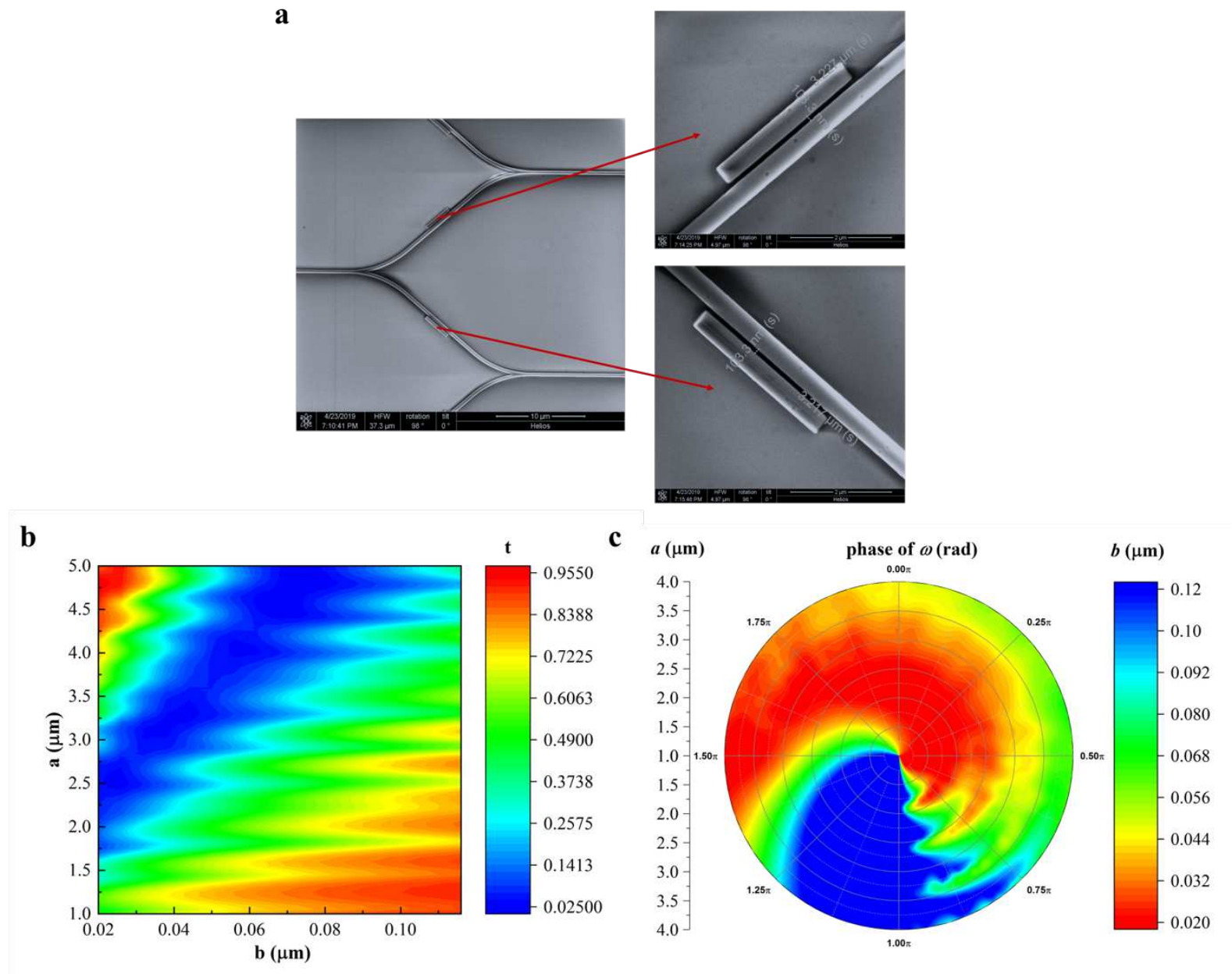

Figure 2 | Weight regulation. a, SEM image of Y-shaped waveguides side-coupled silicon weight modulators. Two arms of the "Y" structure waveguide correspond to two kinds of weights. By regulating the length $a$ of weight modulator and the gap width $b$ between two waveguides: b, The magnitude of weight $\omega$ can be continuously adjusted from 0.03 to 0.95 . $\mathbf{c}$, The phase of weight $\omega$ can be continuously adjusted from 0 to $2 \pi$. 
a

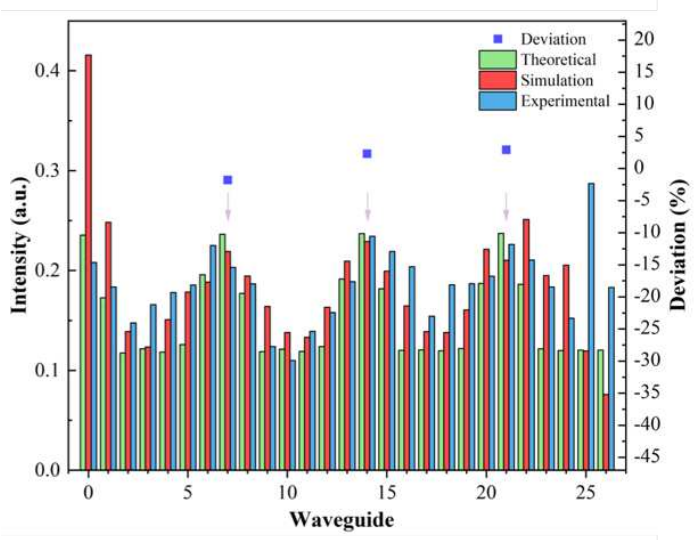

b

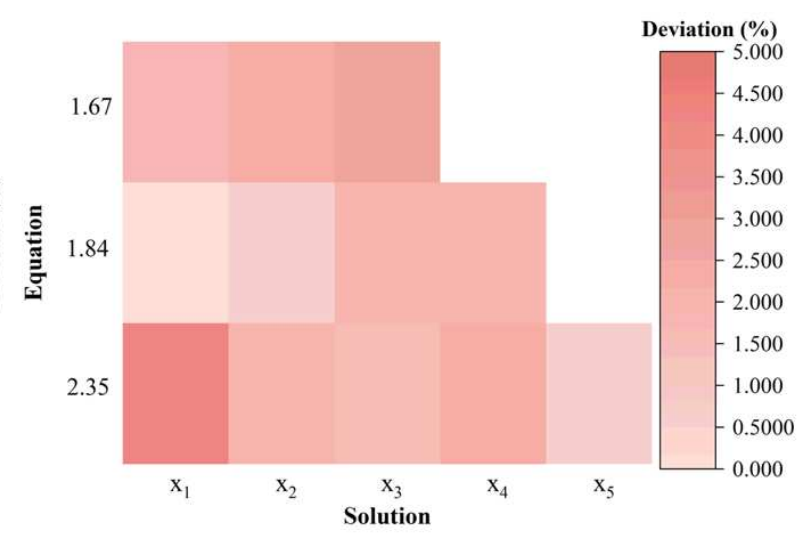

Figure 3 | All-optical transcendental equation solver. a, Output light intensity distribution in the output waveguides $(k=1.67)$. The arrows in the figure correspond to the locations of the solutions. The horizontal axis is the number of discrete waveguides, the vertical axis on the right represents the output signal intensity, and the vertical axis on the left gives the deviation between the experimental output signal and the theoretical value. b, A graphic representation of solution deviation. The horizontal axis labels the individual solutions, and the vertical axis represents three values of the parameter $k$. The shade of the color indicates the magnitude of the deviation. 
a

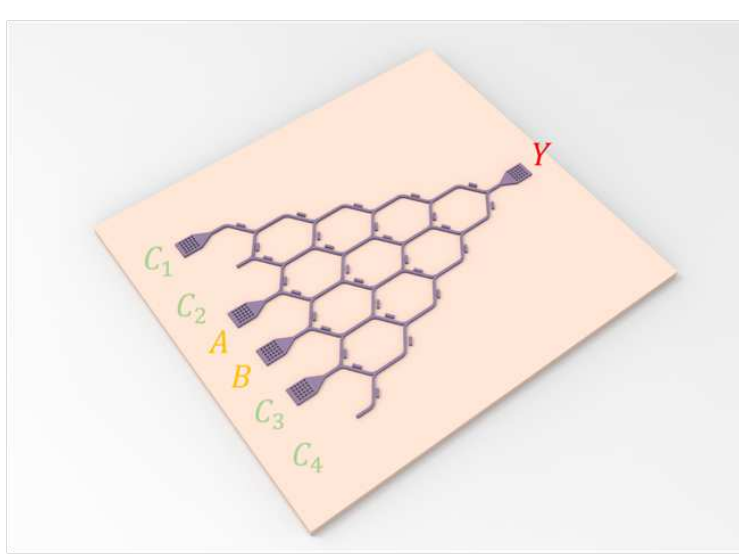

c

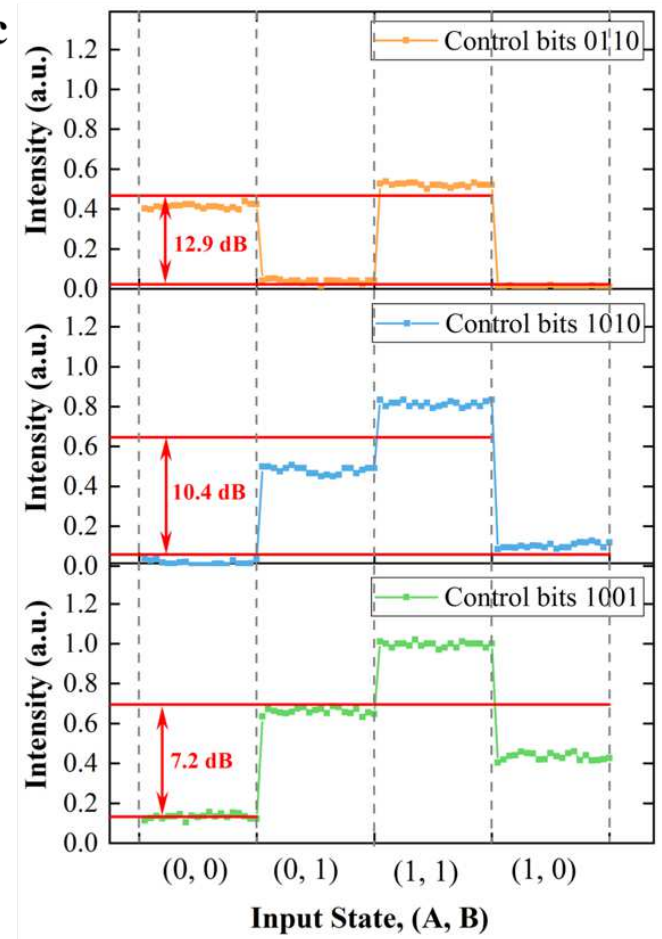

b

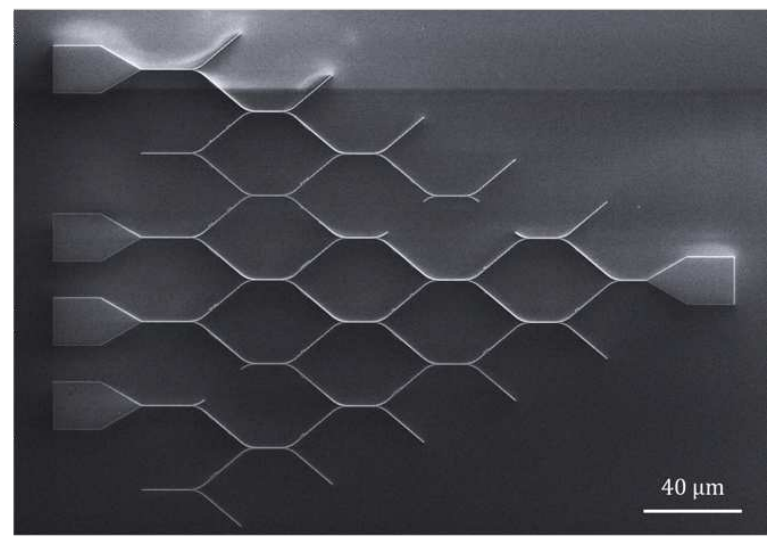

d

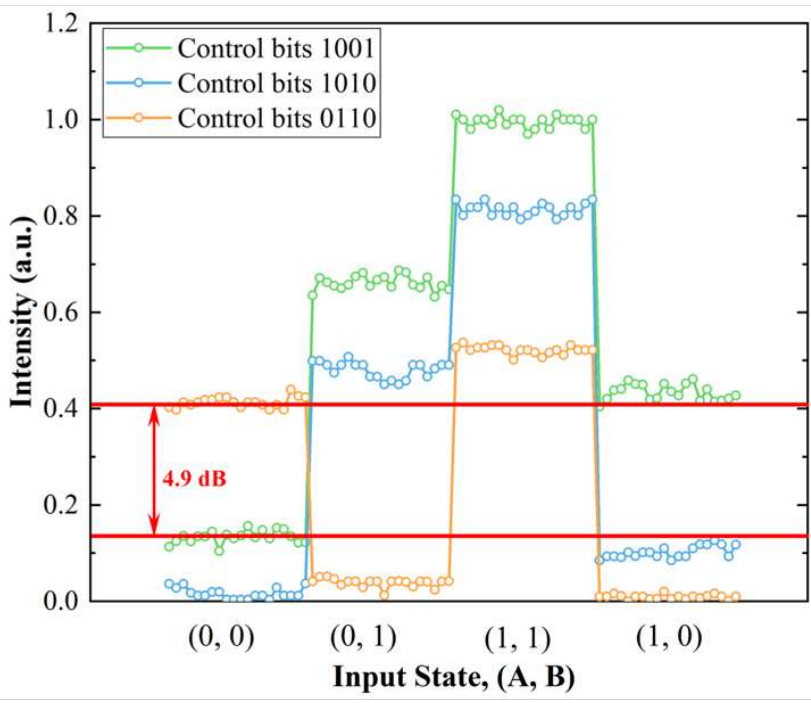

Figure 4 | Multifarious logic gates. a, Schematic diagram of the multifarious logic gate operator. Ports $\mathrm{A}, \mathrm{B}$ are the signal inputs, and ports $C_{1}, C_{2}, C_{3}, C_{4}$ together constitute the control bits, and Y represents the signal output. b, Top-view SEM image of the multifarious logic gate operator. c, 0-1 intensity distribution when the optical $\mathrm{CNN}$ device acts as three different types of logic gates. d, Overlay of three logic function responses in the optical CNN structure. The top red line corresponds to the minimum intensity of "1", and the bottom red line shows the maximum intensity of "0". 
$\mathbf{a}$

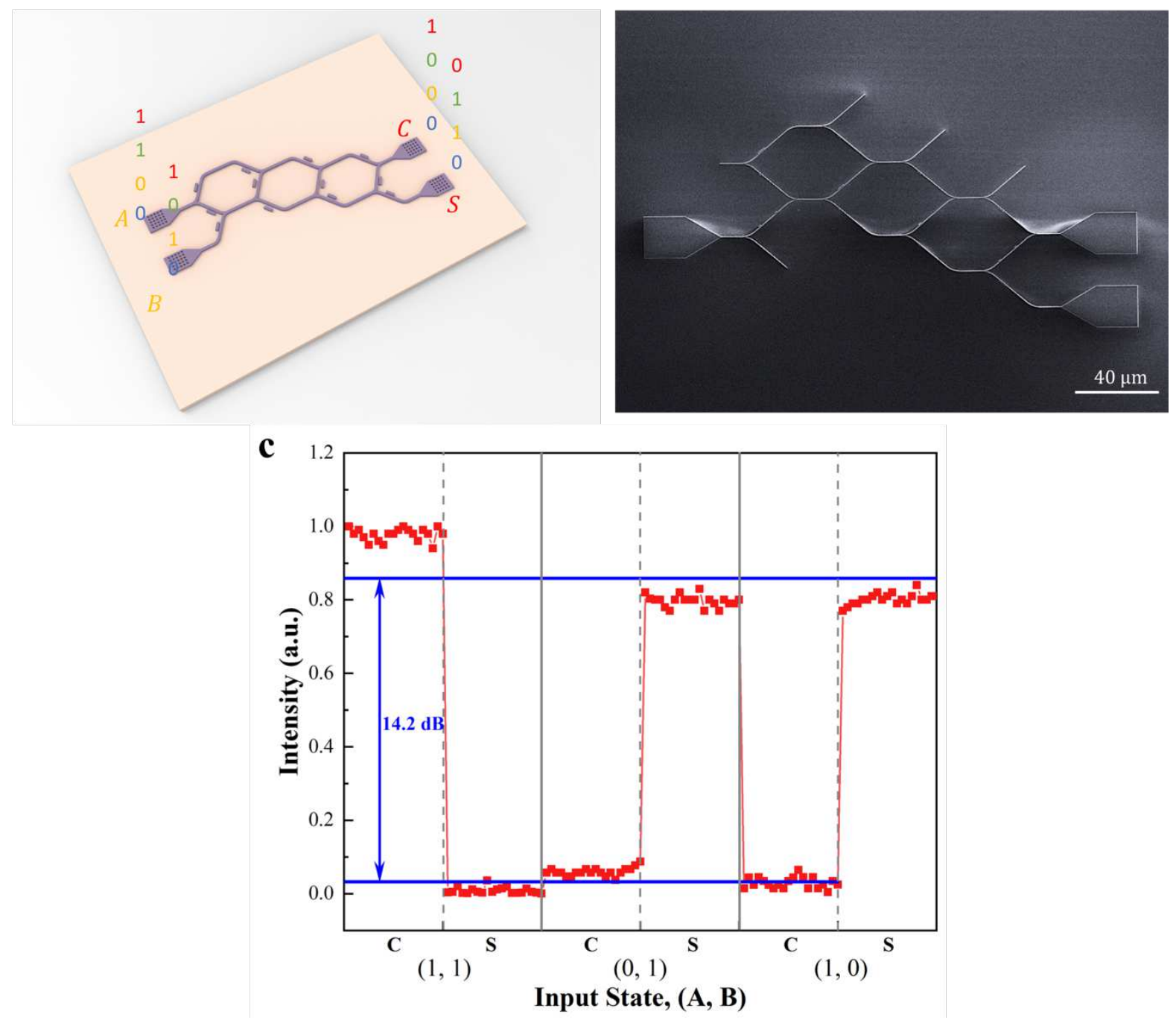

Figure 5 | Half-adder. a, Schematic diagram of the half-adder. Ports A, B are the signal inputs, and $C$ and $S$ represent the Carry and the Sum bit, respectively. b, Top-view SEM image of the half-adder. c, Intensity distribution of Sum bit and carry bit corresponding to three different input signals in the half-adder. The blue lines give the average intensity values of the 0 and 1 logic states. 


\section{Methods}

Theoretical analysis and numerical simulation. PyTorch, a custom package in Python which is used for machine learning popularly, was used to construct the theoretical modeling of our optical neural networks. The calculations were based on 1D CNN used for the equation solver and 2D CNN used for logic devices and half-adder, respectively. Some optimizers were then used in PyTorch, applying stochastic gradient descent (SGD) in the learning process, to compute the parameters in our networks and minimize the loss function related to the model's performance as possible. The simulation results were conducted from finite element method (via the COMSOL Multiphysics commercial software).

Device fabrication. Devices were fabricated leveraging standard silicon microfabrication technologies. A 6\% hydrogen silsesquioxane (HSQ) electron beam resist was spun onto a double-side polished silicon-on-insulator (SOI) wafer and was patterned by an Elionix ELSF125 electron beam lithography (EBL) tool. Development of the resist was performed by immersing the chip into $25 \%$ tetramethylammonium hydroxide solution for 150 seconds. The chip was subsequently etched in an RIE tool (PlasmaTherm Inc.) with chlorine gas at a power of $200 \mathrm{~W}$ and a pressure of $5 \mathrm{mTorr}$. After stripping the electron beam resist in HF, an additional EBL step was conducted to pattern the waveguide grating couplers with ZEP resist on the same EBL tool (etching depth of grating couplers is different from that of transmission waveguide to obtain higher coupling efficiency). The chip was developed in ZED-N50 developer and etched in the same RIE tool under identical conditions. Finally, the resist was stripped by soaking in N-Methyl-2-Pyrrolidone (NMP) overnight.

Optical measurement. Devices were tested on a microspectroscopy measurement system. 
Laser beam from a home-built femtosecond pulse fiber laser system was used as the light source.

The laser central wavelength was $1560 \mathrm{~nm}$ with a repetition rate of $100 \mathrm{MHz}$ and a pulse width of $80 \mathrm{fs}$ (The results are stable in the range of femtosecond pulse wavelength broadening). The signal light with adjustable spot size was focused to the input-coupling port of the sample. The output signal was collected with a long working distance objective lens (Mitutoyo 20, NA = 0.58) and imaged onto a charge coupled device (CCD) camera (Xenics, XS-4407, Belgium).

\section{Acknowledgements}

This work was supported by the National Key Research and Development Program of China (2018YFB2200403); National Natural Science Foundation of China (NSFC) (61775003, 11734001, 91950204, 11527901, 11604378, 91850117); and Beijing Municipal Science \& Technology Commission (Z191100007219001).

\section{Competing interests}

The authors declare no competing interests. 


\section{References:}

1. Salem, R., Foster, M. A. \& Gaeta, A. L. Application of space-time duality to ultrahigh-speed optical signal processing. Adv. Opt. Photonics 5, 274-317 (2013).

2. Marpaung, D., Yao, J. \& Capmany, J. Integrated microwave photonics. Nat. Photonics 13, 80-90 (2019).

3. Willner, A., Khaleghi, S., Chitgarha, M. \& Yilmaz, O. All-Optical Signal Processing. J. Lightwave Technol. 32, 660-680 (2014).

4. Rajaei, R. \& Bakhtavari Mamaghani, S. Ultra-Low Power, Highly Reliable, and Nonvolatile Hybrid MTJ/CMOS Based Full-Adder for Future VLSI Design. IEEE Trans. Device Mater. Reliab. 17, 213-220 (2017).

5. Salahuddin, S., Ni, K. \& Datta, S. The era of hyper-scaling in electronics. Nat. Electron. 1, 442-450 (2018).

6. Smitha, G. S. \& Ravish Aradhya, H. V. mGDI based parallel adder for low power applications. Microsyst. Technol. 25, 1653-1658 (2017).

7. Sun, C. et al. Single-chip microprocessor that communicates directly using light. Nature 528, 534-538 (2015).

8. Ambs, P. Optical Computing: A 60-Year Adventure. Adv. Opt. Technol. 2010, 372652 (2010).

9. Yan, H. et al. Programmable nanowire circuits for nanoprocessors. Nature 470, 240-244 (2011).

10. Ando, M., Kadono, K., Haruta, M., Sakaguchi, T. \& Miya, M. Large third-order optical nonlinearities in transition-metal oxides. Nature 374, 625-627 (1995).

11. Leuthold, J., Koos, C. \& Freude, W. Nonlinear silicon photonics. Nat. Photonics 4, 535-544 (2010).

12. Roy, K., Jaiswal, A. \& Panda, P. Towards spike-based machine intelligence with neuromorphic computing. Nature 575, 607-617 (2019).

13. Xia, Q. F. \& Yang, J. J. Memristive crossbar arrays for brain-inspired computing. Nat. Mater. 18, 309-323 (2019).

14. Xing Lin, Y. R., Nezih T. Yardimci, Muhammed Veli, Yi Luo, Mona Jarrahi, Aydogan Ozcan. All-optical machine learning using diffractive deep neural networks. Science 361, 1004-1008 (2018).

15. Yan, T. et al. Fourier-space Diffractive Deep Neural Network. Phys. Rev. Lett. 123, 023901 (2019).

16. Shen, Y. et al. Deep learning with coherent nanophotonic circuits. Nat. Photonics 11, 441-446 (2017).

17. Feldmann, J., Youngblood, N., Wright, C. D., Bhaskaran, H. \& Pernice, W. H. P. All-optical spiking neurosynaptic networks with self-learning capabilities. Nature 569, 208-214 (2019).

18. Brunner, D., Soriano, M. C., Mirasso, C. R. \& Fischer, I. Parallel photonic information processing at gigabyte per second data rates using transient states. Nat. Commun. 4, 1364 (2013).

19. Antonik, P., Marsal, N., Brunner, D. \& Rontani, D. Human action recognition with a large-scale brain-inspired photonic computer. Nature Machine Intelligence 1, 530-537 (2019).

20. Nasim Mohammadi Estakhri, B. E., Nader Engheta. Inverse-designed metastructures that solve equations. Science 363, 1333-1338 (2019).

21. Ballarini, D. et al. All-optical polariton transistor. Nat. Commun. 5, 1778 (2014).

22. Lu, C., Hu, X., Yang, H. \& Gong, Q. All-optical logic binary encoder based on asymmetric plasmonic nanogrooves. Appl. Phys. Lett. 103, 121107 (2013).

23. Wang, F. F. et al. Nanoscale on-chip all-optical logic parity checker in integrated plasmonic circuits in optical communication range. Sci. Rep. 6, 24433 (2016).

24. Liu, C. S. et al. Small footprint transistor architecture for photoswitching logic and in situ memory. Nat. Nanotechnol. 14, 662-667 (2019). 


\section{Figures}

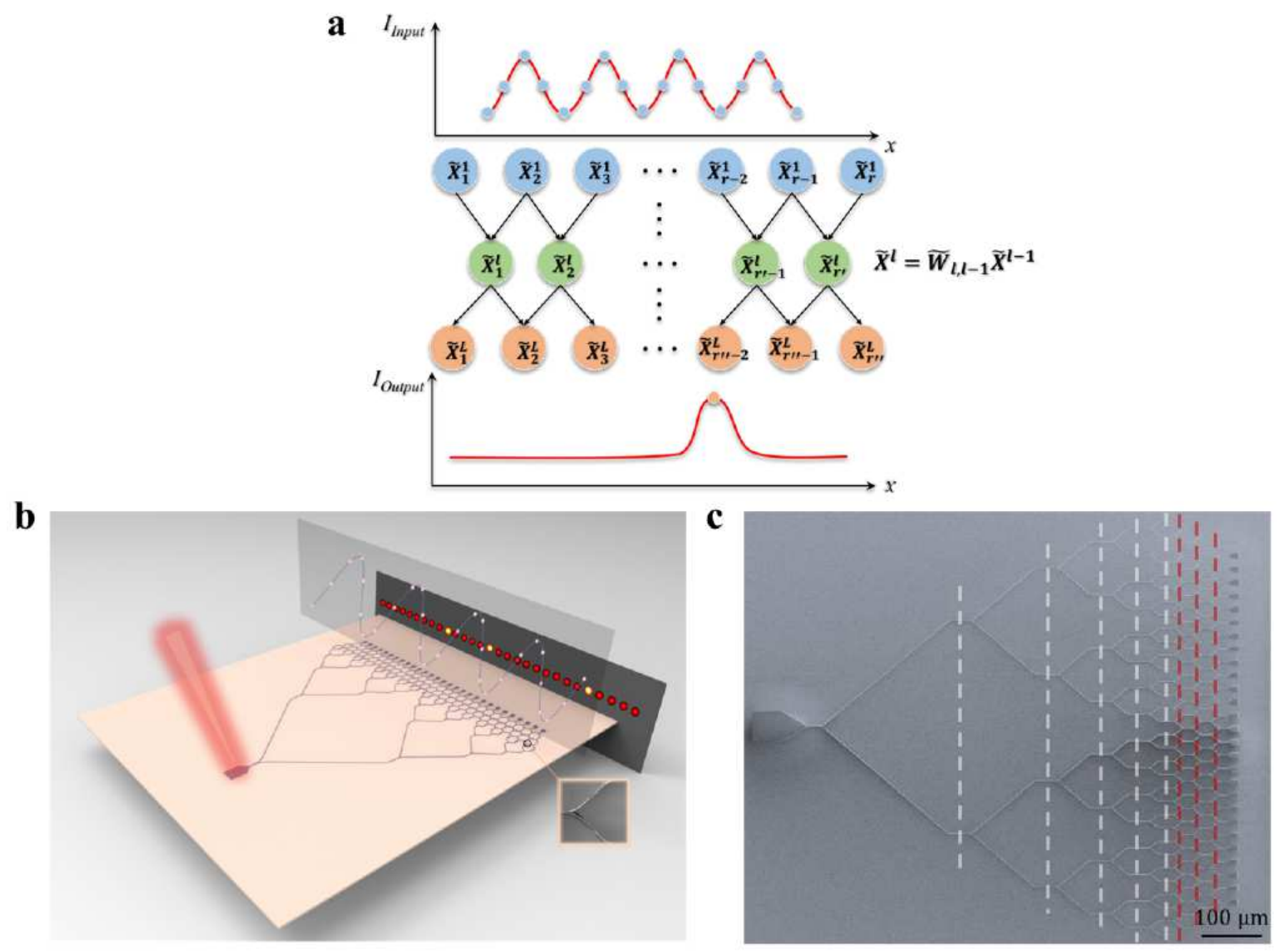

\section{Figure 1}

General architecture of the all-optical computing framework. a, The CNN architecture showing the

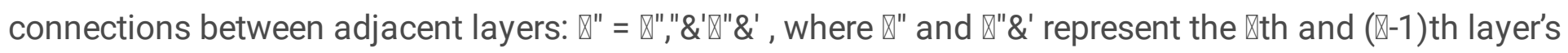

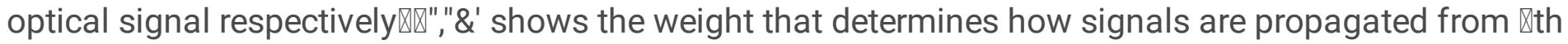

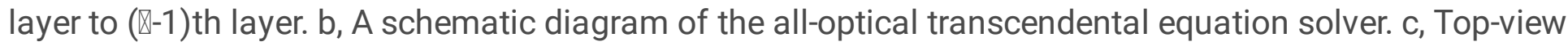
SEM image of the alloptical transcendental equation solver, where the scale bar is $100 \mu \mathrm{m}$. Here, the white dotted lines mark the five layers for waveform discretization, and the red dotted lines separate the three layers of the optical CNN structure. 
a

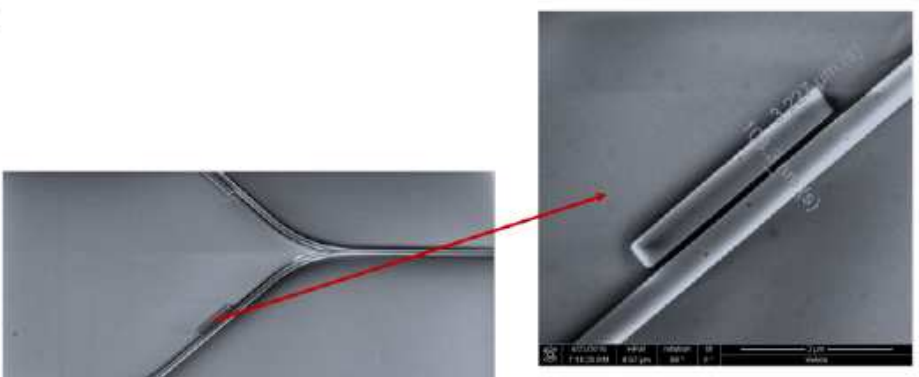

b

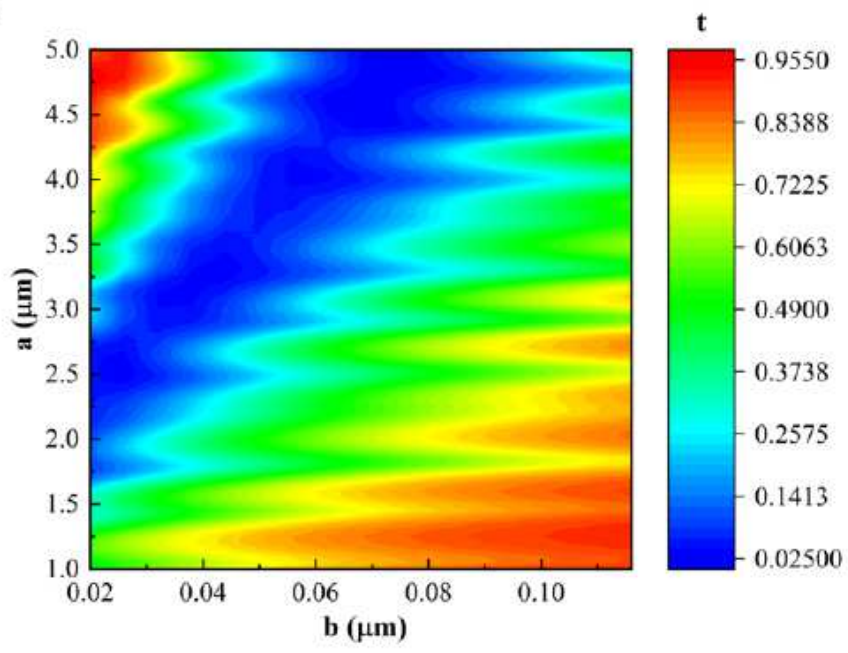

c $a(\mu \mathrm{m})$
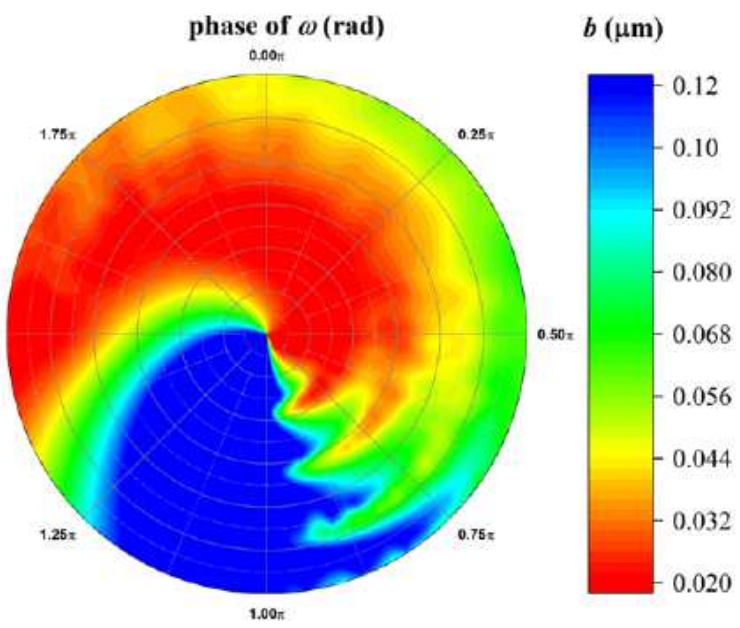

Figure 2

Weight regulation. a, SEM image of Y-shaped waveguides side-coupled silicon weight modulators. Two arms of the " $Y$ " structure waveguide correspond to two kinds of weights. By regulating the length $\nabla$ of weight modulator and the gap width $\otimes$ between two waveguides: $b$, The magnitude of weight $\omega$ can be continuously adjusted from 0.03 to 0.95 . c, The phase of weight $\omega$ can be continuously adjusted from 0 to $2 \pi$. 
$\mathbf{a}$

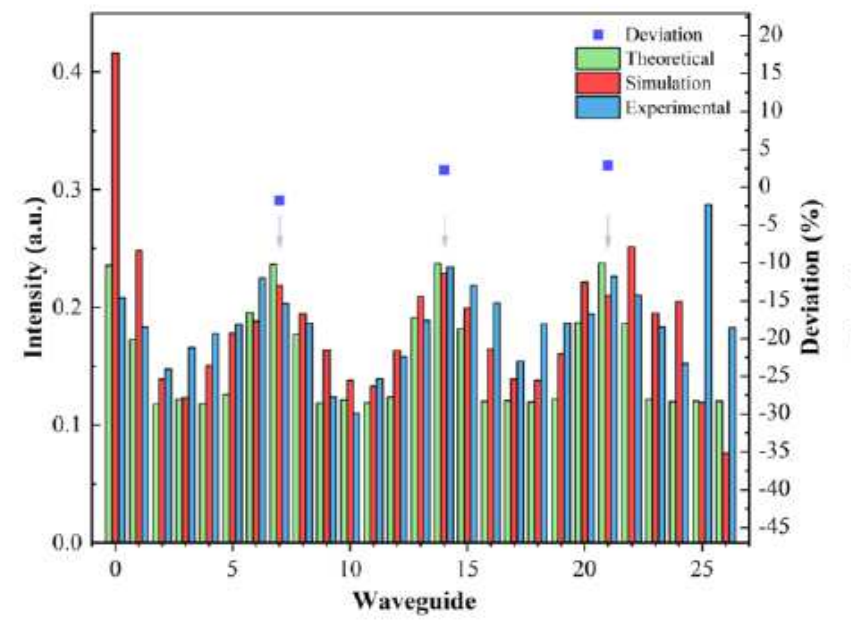

b

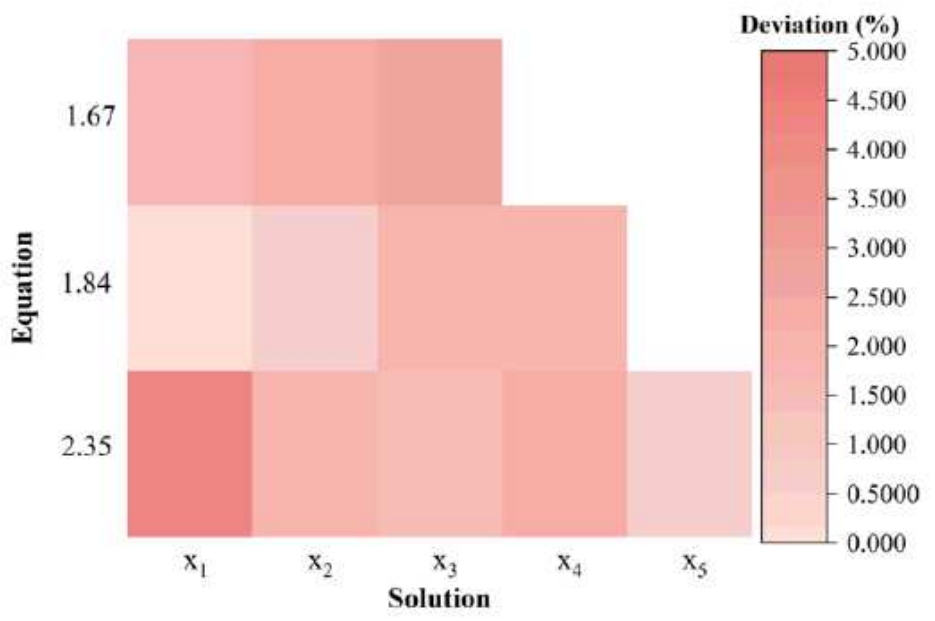

\section{Figure 3}

All-optical transcendental equation solver. a, Output light intensity distribution in the output waveguides ( $\square$ $=1.67)$. The arrows in the figure correspond to the locations of the solutions. The horizontal axis is the number of discrete waveguides, the vertical axis on the right represents the output signal intensity, and the vertical axis on the left gives the deviation between the experimental output signal and the theoretical value. b, A graphic representation of solution deviation. The horizontal axis labels the individual solutions, and the vertical axis represents three values of the parameter $\nabla$. The shade of the color indicates the magnitude of the deviation. 
a

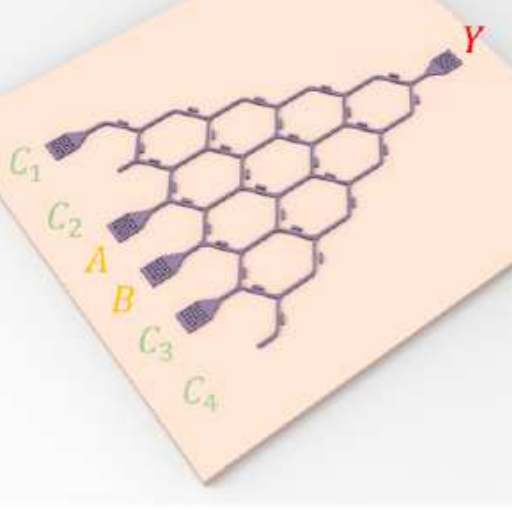

c

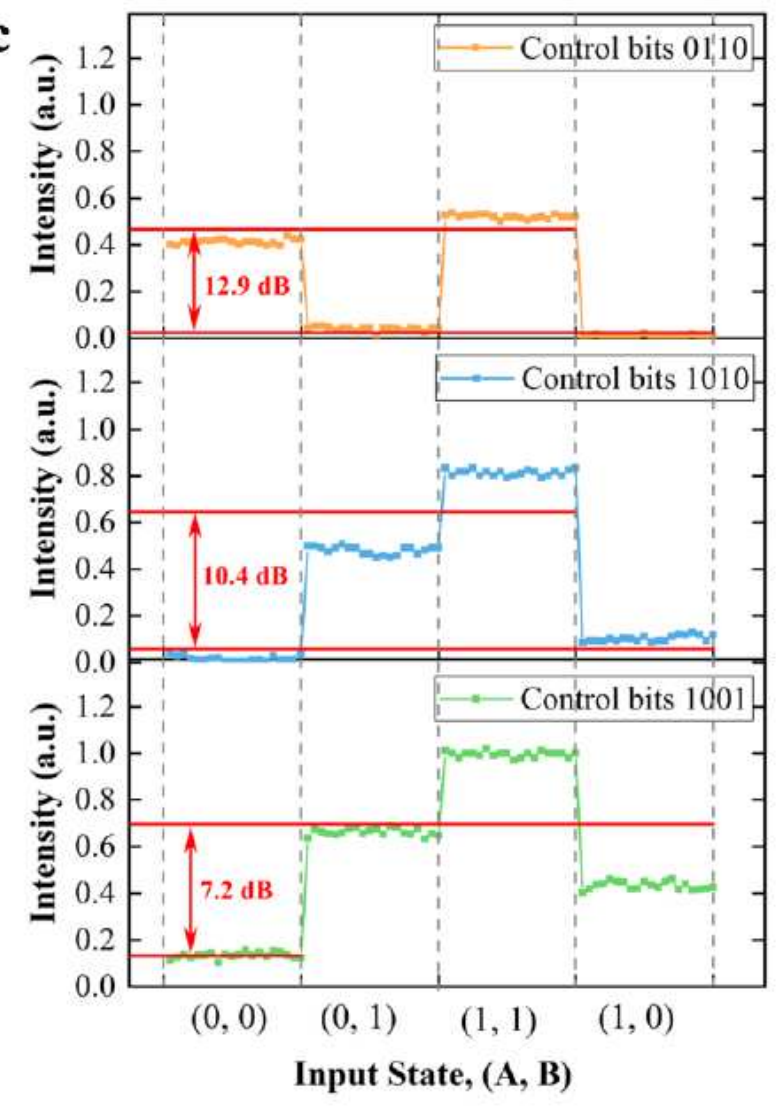

b

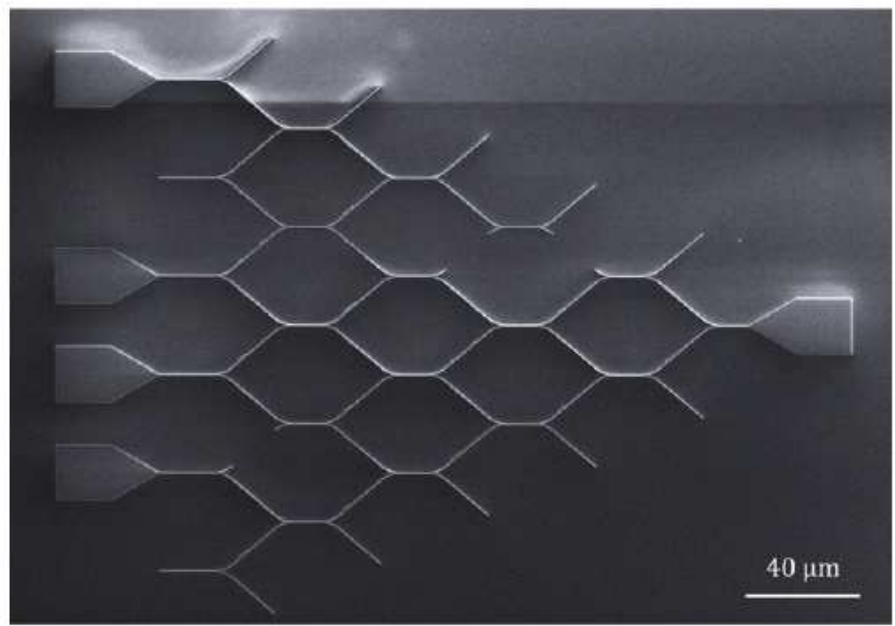

d

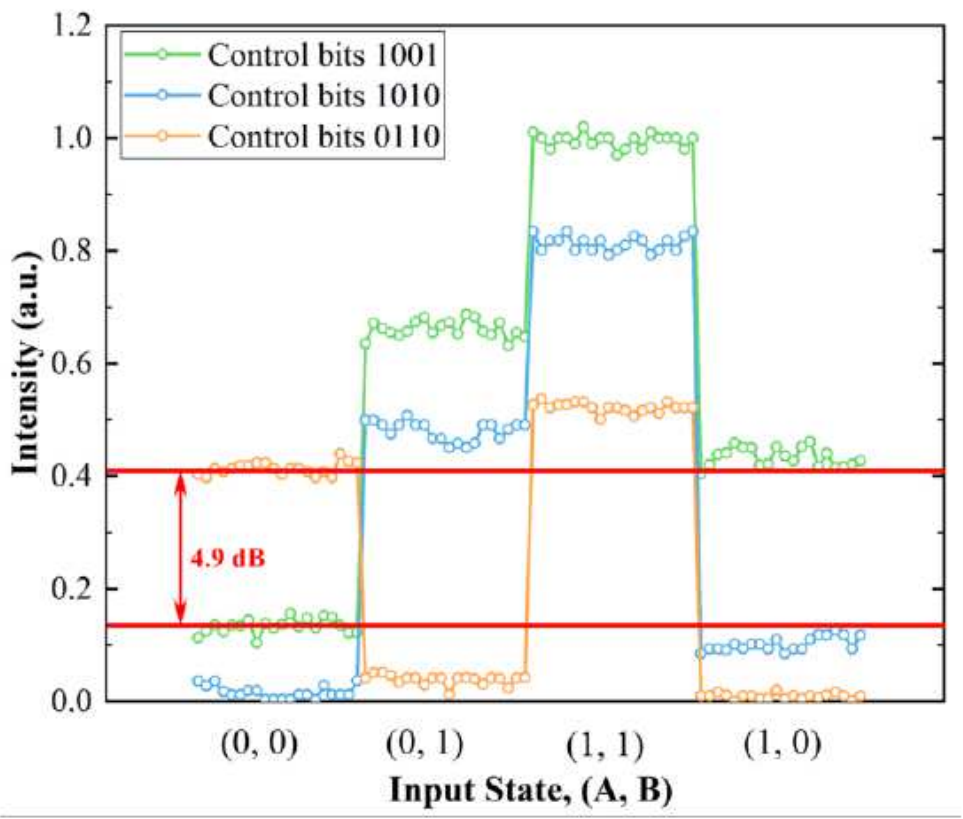

\section{Figure 4}

Multifarious logic gates. a, Schematic diagram of the multifarious logic gate operator. Ports A, B are the signal inputs, and ports $\nabla^{\prime}, \varangle \mathbf{f}, \mathbb{} \mathbf{g}$, $\backslash \backslash$ together constitute the control bits, and $Y$ represents the signal output. b, Top-view SEM image of the multifarious logic gate operator. c, 0-1 intensity distribution when the optical CNN device acts as three different types of logic gates. $d$, Overlay of three logic function responses in the optical CNN structure. The top red line corresponds to the minimum intensity of " 1 ", and the bottom red line shows the maximum intensity of " 0 ". 
a

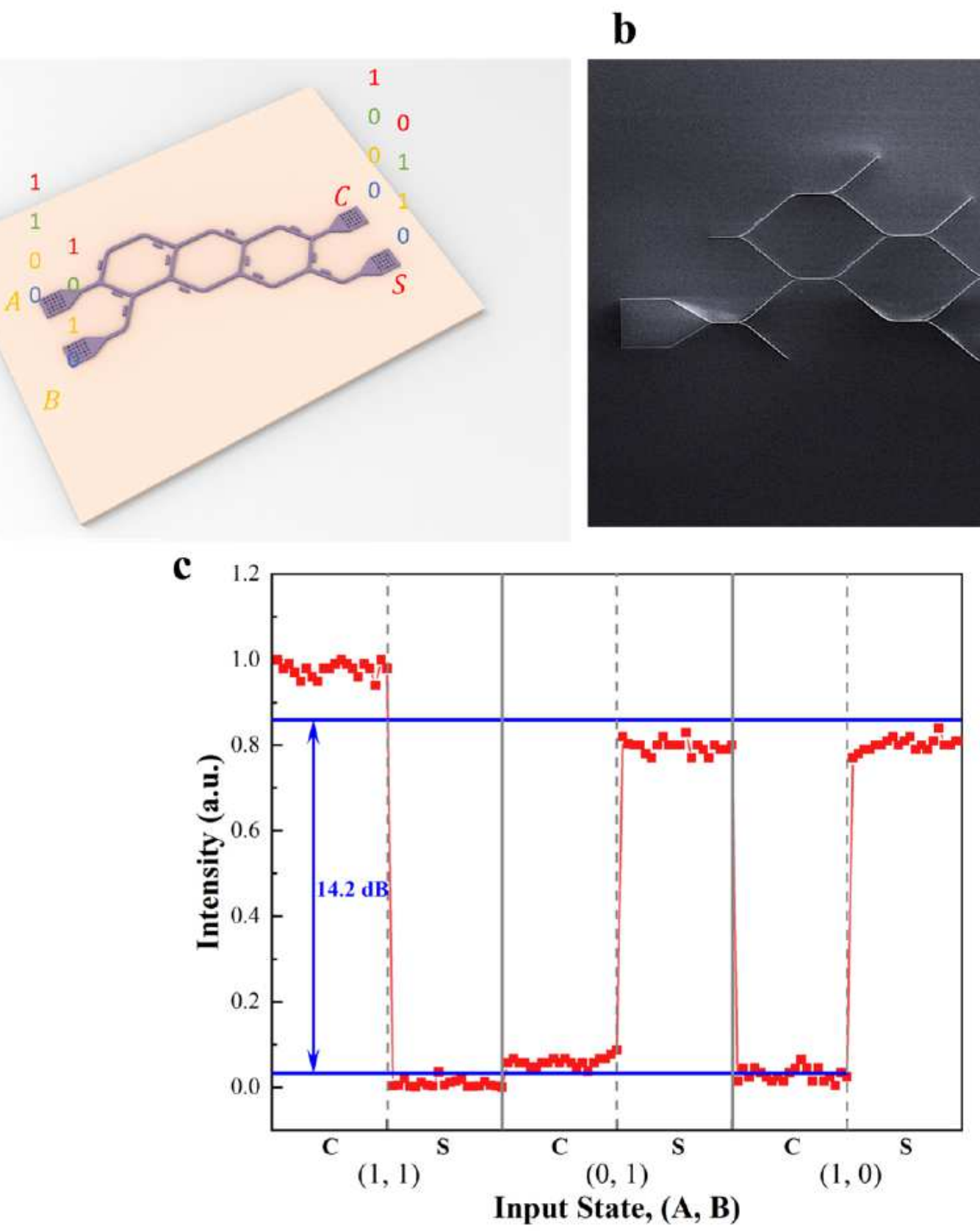

Figure 5

Half-adder. a, Schematic diagram of the half-adder. Ports $A, B$ are the signal inputs, and $\triangle$ and $\nabla$ represent the Carry and the Sum bit, respectively. b, Top-view SEM image of the half-adder. c, Intensity distribution of Sum bit and carry bit corresponding to three different input signals in the half-adder. The blue lines give the average intensity values of the 0 and 1 logic states.

\section{Supplementary Files}

This is a list of supplementary files associated with this preprint. Click to download.

- Supplementarylnformation.pdf 